\title{
Gene silencing of E-selectin block recruitment of endothelial progenitor cell to vascular endothelium under flow
}

\author{
Sunil Sharma, Masayuki Yoshida
}

Life Science and Bioethics Research Center, Tokyo Medical and Dental University, Tokyo, Japan. Email: masavasc@tmd.ac.jp

Received 16 February 2010; revised 20 March 2010; accepted 25 March 2010.

\begin{abstract}
Short interfering RNA (siRNA) is a powerful technique that can suppress gene expression in a variety of cells including mammalian cells. Endothelial progenitor cells (EPCs) are bone marrow-derived haematopoietic progenitor cells that have been implicated in vasculogenesis. We demonstrated for the first time that gene silencing of endothelial E-selectin using siRNA transfection in human umbilical vein endothelial cells (HUVECs) causes inhibition of EPC adhesion under flow conditions. Fluorescence immunobinding assay analysis showed that significant reduction of E-selectin surface expression in $\mathrm{HU}$ -

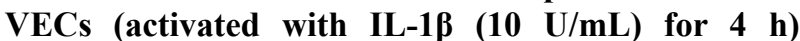
transfected with siRNA against E-selectin, but not in HUVECs transfected with $L a c Z$ siRNA (control). An EPC adhesion assay under flow conditions (shear stress $=1.0 \mathrm{dyne} / \mathrm{cm}^{2}$ ) then demonstrated that $\mathrm{HU}$ VECs transfected with E-selectin siRNA supported significantly less adhesion of EPCs than those HUVECs treated with control siRNA and no siRNA after activation by IL-1 $\beta(p<0.05)$. Our experiments have shown the importance of E-selectin in EPC adhesion to HUVECs and the potential utility of gene silencing of E-selectin in EPC recruitment.
\end{abstract}

Keywords: Endothelial Progenitor Cell; E-Selectin; Rnai

\section{INTRODUCTION}

The vascular endothelium is a vital border zone between the circulatory system and the tissues it supplies. It is a monolayer of endothelial cells (EC) that has pivotal roles in coagulation, inflammation, vasodilatation and vasoconstriction through substances such as nitric oxide and prostaglandins [1].

Recently identified vascular progenitor cells from bone marrow (BM) and non-BM origin have been shown to contribute to neovessel formation [2]. Upon inflammatory conditions, the vascular endothelium was acti- vated to capture progenitor cells in regions where endothelium regeneration is needed.

Though ischemia is believed to be the physiological stimulus for EPC mobilisation from the bone marrow, the mechanisms of EPC recruitment are less well understood.

Little is understood concerning the molecular mechanisms of EPC adhesion to endothelial cells. In contrast, extensive studies have been conducted regarding leukocyte-endothelial interactions. Previous experiments have demonstrated that P-selectin and E-selectin are involved in leukocyte rolling, whilst intercellular adhesion molecule-1 (ICAM-1) is associated with leukocyte firm adhesion [3]. The molecular players involved in leukocyte transmigration have been found to be molecules such as platelet-endothelial-cell adhesion molecule-1 (PECAM-1), junctional adhesion molecules (JAMs), vascular endothelial-cadherin (VE-cadherin) and CD99 [4].

Chavakis et al had implicated roles for $\beta 2$-integrins in the homing and revascularisation of EPCs [5]. Vajcokzy et al had carried out in vivo experiments with mice to demonstrate that E-selectin is involved in adhesion of embryonic EPCs [6].

We recently reported an important role of endothelial E-selectin in mediating endothelial progenitor cell (EPC) recruitment [7].

In this study, we used a flow chamber system to mimic the in vivo circulation. The major advantage of this set-up is that conditions can be much more tightly controlled, so that the effect of individual molecules can be better ascertained than with an in vivo procedure.

RNA inhibition has allowed us to silence genes for a variety of purposes. Short interfering RNA (siRNA) is a useful technique to define the function of a given molecule [8].

In this study we look at the role of E-selectin in EPC adhesion to endothelial cells using double stranded short interfering RNA (siRNA), in conjunction with the in vitro flow assay system. Knowledge of this study will allow us to better understand the process of EPC re- 
cruitment to endothelial cells, which will give greater insight into the process of vasculogenesis and its clinical implications.

\section{MATERIALS AND METHOD}

\subsection{Cell Culture and Reagents}

Human umbilical vein endothelial cells (HUVECs) were cultured on $0.1 \%$ gelatin-coated tissue culture dishes as described previously $[9,10]$. The following antibodies were used in this study; H18/7 [11] (anti-human E-selectin mAb), Hu5/3 [12] (anti-human ICAM-1 $\mathrm{mAb})$. Recombinant human IL-1 $\beta$ was a gift from Biogen Inc (Cambridge, MA). The HL60 cell lines were obtained from the American Type Culture Collection and cultured in RPMI-1640 containing 10\% FCS. For use in the flow-chamber apparatus, HUVECs were plated onto $22 \mathrm{~mm}$ fibronectin-coated glass coverslips as has been described previously $[13,14]$.

Preparation of endothelial progenitor cells was previously described in detail [7].

In brief, $25 \mathrm{~mL}$ of the blood was collected from human volunteers, to which $15 \mathrm{~mL}$ Histopaque ${ }^{\circledR}-1077$ solution (Sigma-Aldrich Japan, KK) was added. After centrifugation for 20 minutes at $1600 \mathrm{rpm}$, the upper layer was collected and diluted in Dulbecco's phosphate buffered saline, then add $2 \mathrm{mM}$ EDTA (DPBSE, Sigma) and centrifuged at $2000 \mathrm{rpm}$ for 5 minutes. The residual pellet was resuspended in $10 \mathrm{~mL}$ ammonium chloride solution (Stem Cell Technologies Inc) to lyse red blood cells and centrifuged at $1000 \mathrm{rpm}$ for 10 minutes. The pellet was resuspended in Endothelial Cell Basal Medium $\left(\right.$ EBM-2, Clonetics $\left.{ }^{\mathbb{B}}\right)$ and cultured in each well of a C-6 plate (coated with fibronectin). Media exchange of the EPC culture was carried out after 4 days, and EPCs were used for flow assay after 7 days. All experimental procedures were approved by the Experimental Research Review Committee of the Tokyo Medical and Dental University (No. 0090014).

\subsection{Flow Assay}

The parallel-plate flow chamber that was used in this study has been described previously in more detail $[15,16]$. In summary, the chamber was composed of 2 aluminium steel plates separated by a $200 \mu \mathrm{m}$ thick silastic gasket, and the flow channel was formed by removal of a $5 \times 20 \mathrm{~mm}$ rectangular section from the gasket. Defined levels of flow were applied to the HUVEC monolayer by drawing the flow media (see above) through the channel with a syringe pump (model 44 Harvard Apparatus). A plastic heating plate (Tokai Hit Co) was attached to the stage of an inverted microscope (IX50, Olympus) to maintain the temperature at $37^{\circ} \mathrm{C}$. Using this set-up, the channel flow could be modelled as a two-dimensional fully developed laminar flow with a simple parabolic velocity profile.

HUVEC monolayers on coverslips were stimulated for $4 \mathrm{~h}$ using IL-1 $\beta(10 \mathrm{U} / \mathrm{mL})$, mixed with RPMI and $1 \%$ FBS, then positioned in the flow chamber. The monolayers were perfused for 5 minutes with perfusion medium (flow media), then examined carefully to establish the monolayer as confluent. HL60 cells or EPCs were then diluted in the flow media to $1 \times 10^{6}$ cells $/ \mathrm{mL}$. The cells were then drawn through the chamber at controlled flow rates to achieve wall shear stresses of 1.0 and 2.0 dyne $/ \mathrm{cm}^{2}$ for 10 minutes.

\subsection{Fluorescent Immunobinding Assay (FIA)}

HUVEC monolayers in 96-well plates were incubated on ice with the indicated primary antibody in RPMI $1 \%$ FCS at $10 \mu \mathrm{g} / \mathrm{mL}$ for 45 minutes. These wells were then washed three times with RPMI $/ \%$ FCS, after which they were incubated with a FITC-conjugated goat anti-mouse polyclonal $\left.\mathrm{F}(\mathrm{ab})_{2}\right)_{2}$ antibody (Caltag Laboratories) diluted 1:100 in DPBS on ice. After 45 minutes, the wells were washed with DPBS $/ 20 \%$ FCS twice, followed by teo washes with DPBS only. Cells were lysed with $0.01 \% \mathrm{NaOH}$ in $0.1 \%$ SDS. The fluorescence was then measured using a CytoFluor II (Perspective Biosystems) fluorescent plate reader set at 485 (excitation)/ 535 (emission).

\section{4. siRNA Transfection of E-Selectin}

Short interfering RNAs (siRNAs) were designed to target the coding sequence of human E-selectin cDNA. The target sequences were directed as described previously ${ }^{8}$. Briefly, the sequences were targeted to the single-strand region consistent with the predicted secondary RNA structure and sequences of the form $(\mathrm{AA} / \mathrm{CA}) \mathrm{N}_{19}$ with GC contents of less than $70 \%$ were isolated from this region. Nineteen RNA nucleotides followed by TT/TG were selected, then chemically synthesised, and finally gel-purified.

In order to anneal the single stock strands of RNAi (JbioS) $20 \mu \mathrm{L} 50 \mu \mathrm{M}$ sense, $20 \mu \mathrm{L} 50 \mu \mathrm{M}$ antisense and $10 \mu \mathrm{L} 10 \mathrm{mM} \mathrm{MgCl}{ }_{2}$ in DEPC-PBS was mixed together, giving a dsRNA concentration of $20 \mu \mathrm{M}$. This was then heated at $95^{\circ} \mathrm{C}$ for 2 minutes, $70^{\circ} \mathrm{C}$ for 1 minute, $20^{\circ} \mathrm{C}$ for 30 minutes, and then $4^{\circ} \mathrm{C}$ before being stored at $-80^{\circ} \mathrm{C}$. Solutions of $120 \mu \mathrm{L}$ Optimem ${ }^{\circledR}$ (Invitrogen), $6 \mu \mathrm{L}$ double-stranded siRNA for E-selectin (siE-01) (or control using $L a c Z$ (scE-01) or no siRNA (i.e. Lipofectin alone)), $120 \mu \mathrm{L} \mathrm{Optimem}^{\circledR}$ (Invitrogen) and $5 \mu \mathrm{L}$ Lipofectin $^{\circledR}$ (Invitrogen) were prepared, and left for 30 minutes then combined and again left for 30 minutes. Optimem ${ }^{\mathbb{B}}$ was then added to make up a total volume of $1.2 \mathrm{~mL}$. This solution was then added to HUVEC monolayers prewashed with Optimem ${ }^{\circledR}$, then incubated for $4 \mathrm{~h}$ at $37^{\circ} \mathrm{C}$, 
after which the HUVEC media was changed to EBM-2. The cells were used $24 \mathrm{~h}$ after transfection.

\subsection{Western Blot}

Western blot analysis was performed using lysates prepared from HUVEC, as described previously [7]. An equal amount of protein $(10 \mu \mathrm{g})$ from each condition was subjected to $5-20 \%$ SDS-PAGE. I mmunoreactive proteins were detected using an enhanced chemiluminescence (ECL) kit (Amersham Bioscience).

\subsection{Statistical Analysis}

Results are presented as mean \pm standard deviation as indicated. Two-tailed Student's $t$ tests were performed using Microsoft Excel to analyse data. Probability values represent the results of these $t$ tests with a value of $\mathrm{p}<$ 0.05 considered statistically significant.

\section{RESULTS}

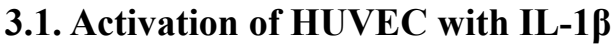

First, we tried an adhesion assay using monocytic HL60 cells over an activated HUVEC monolayer to validate our assay system. As shown in Figure 1, we found a time-dependent increase of HL60 rolling and adhesion when stimulated with IL-1 $\beta(10 \mathrm{U} / \mathrm{mL})$, with a peak activation after $4 \mathrm{~h}$ stimulation. $2 \mathrm{~h}$ IL-1 $\beta$ stimulation led to a dramatic increase in adhesion $(13.3 \pm 9.92$ cells $/ \mathrm{HPF}$, $\mathrm{p}<0.0005)$ and rolling $(4.8 \pm 3.34, \mathrm{p}<0.0005)$ of HL60 cells when compared to those HUVECs without activation (adhesion, $1.8 \pm 1.78$; rolling, $0 \pm 0$ ). $4 \mathrm{~h} \mathrm{IL-1 \beta}$ stimulation led to a further increase in adhesion $(25.8 \pm$ 12.1 cells/HPF, $\mathrm{p}<0.00001)$ and rolling $(3 \pm 2.83$, $\mathrm{p}<$ 0.03 ) of HL60 cells. IL-1 $\beta$ stimulation for $6 \mathrm{~h}$ caused an increase in adhesion of HL60 cells greater than that with $2 \mathrm{~h} \mathrm{IL}-1 \beta$ stimulation but not as significant as that with 4 $\mathrm{h}$ IL-1 $\beta$ stimulation, when compared to the control sample $(6 \mathrm{~h}, 16.5 \pm 4.90$ cells vs $0 \mathrm{~h}, 1.8 \pm 1.78$ cells, $\mathrm{p}=$ $\left.5.62 \times 10^{-8}\right)$. We then checked the surface expression of adhesion molecules after IL-1 $\beta$ activation using fluorescent immunobinding assay (FIA), as shown in Figure 2. To check the validity of the FIA analysis, intensity of negative controls (no primary antibody) and positive controls (W6/32, anti-HLA Class I antibody) were examined (negative control: $0 \mathrm{~h}, 4.8 \pm 3.19 \mathrm{RFU}$ vs $4 \mathrm{~h}$, $5.6 \pm 3.29$ RFU, $p=0.71$; positive control: $0 \mathrm{~h}, 17 \pm 2$ RFU vs $4 \mathrm{~h}, 21.2 \pm 2.59 \mathrm{RFU}, \mathrm{p}=0.02)$. E-selectin expression (detected using $\mathrm{H} 18 / 7 \mathrm{Ab}$ ) was significantly upregulated after $4 \mathrm{~h}$ IL-1 $\beta$ stimulation compared to no stimulation (4 h, $25.2 \pm 1.79 \mathrm{RFU}$ vs $0 \mathrm{~h}, 9 \pm 2.92 \mathrm{RFU}$, $\mathrm{p}=5.52 \times 10^{-6}$ ). ICAM-1 expression (detected using $\mathrm{Hu} 5 / 3 \mathrm{Ab}$ ) was also significantly upregulated after $4 \mathrm{~h}$ IL-1 $\beta$ stimulation $(4 \mathrm{~h}, 23.4 \pm 3.05 \mathrm{RFU}$ vs 0 h, $9.2 \pm$ $0.84 \mathrm{RFU}, \mathrm{p}=8.23 \times 10^{-6}$ ).

\subsection{Adhesion of EPC to Activated HUVEC under Flow}

Based on the preliminary experiment using HL-60 cells

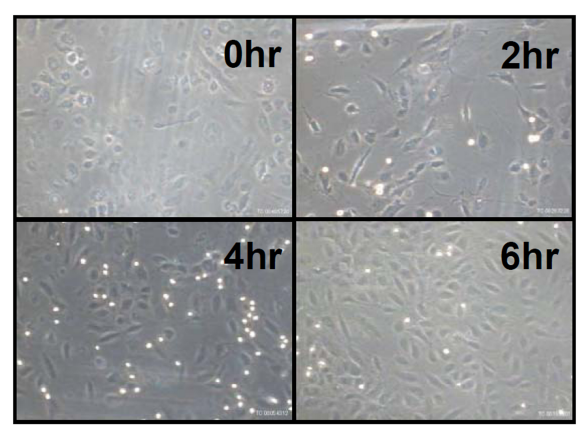

(a)

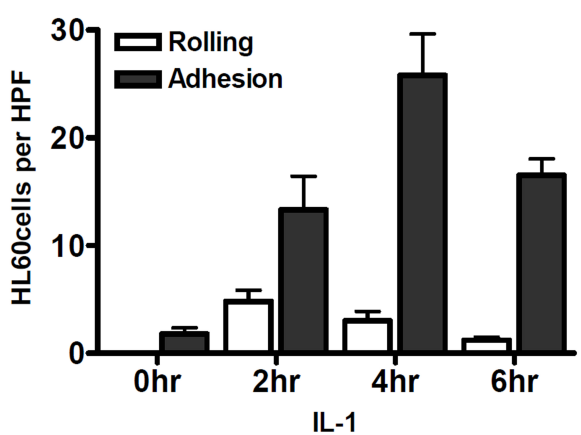

(b)

Figure 1. IL-1 $\beta$ induces a time-dependent rolling and adhesion of HL60 cells to HUVEC. (a) Representative micrographs taken from video recording during the flow assay. HL60 cells (white particles) were perfused over a HUVEC monolayer (grey background) that had been subjected to activation by IL-1 $\beta(50 \mathrm{U} / \mathrm{mL})$ for four different time periods; (b) The number of rolling and adherent HL60 cells were measured as described in Methods. (* $=p<0.05$ vs 0 h IL-1 $\beta$ ); data based on 10 fields of observation for each condition.

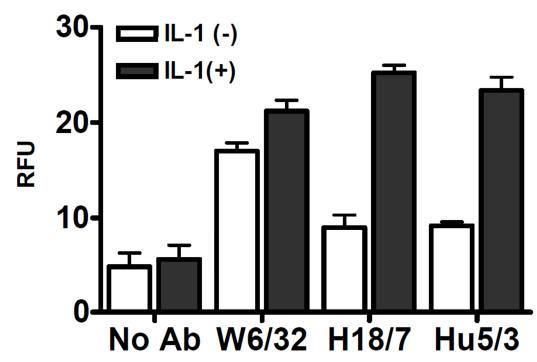

Figure 2. Cell surface expression of E-selectin and ICAM-1 in HUVEC after IL-1 $\beta$ stimulation. The expression intensity of each of the adhesion molecules was determined using a fluorescent immunobinding assay as described in Methods. $*^{*}=\mathrm{p}$ $<0.05$ vs corresponding $0 \mathrm{~h} \mathrm{IL-1 \beta}$ ); data based on 5 repeats for each condition. 
(Figure 1), we used a condition of IL-1 $\beta(10 \mathrm{U} / \mathrm{mL})$ for $4 \mathrm{~h}$ in our following experiments. When EPCs, cultured ex vivo for 7 days, were perfused over activated HUVEC monolayer, adhesion of EPCs was dramatically increased compared to the control HUVEC monolayer $(4 \mathrm{~h}, 4.4 \pm 1.50$ cells vs $0 \mathrm{~h}, 1.3 \pm 0.9$ cells, $\mathrm{p}=8.97 \times$ $10^{-5}$ ), as shown in Figure 3. There was however no rolling of EPCs observed both in the control and $4 \mathrm{~h} \mathrm{IL-1} \beta$ stimulation.

\subsection{Gene Silencing of E-Selectin in HUVEC}

Having demonstrated that $4 \mathrm{~h}$ of IL- $1 \beta$ stimulation was optimal for adhesion, we looked at the effects of silencing E-selectin, using siRNA transfection, on the rolling and adhesion of EPCs. siRNA transfection on HUVEC monolayers was carried out $24 \mathrm{~h}$ prior to the adhesion assay under flow conditions (the time point was determined to optimize the effect of siRNAs in HUVECs, as previously described [8]). We utilized FIA assay to demonstrate the level of E-selectin in these cells. As demonstrated in Figure 4, positive controls (W6/32, anti-HLA Class I antibody) exhibited no significant difference between siRNA treatment groups, enhancing the validity of these results. However, there was a significant reduction of E-selectin surface expression in the E-selectin siRNA - treated HUVECs when compared to the LacZ siRNA -treated HUVECs after $4 \mathrm{~h} \mathrm{IL-1} \beta$ stimulation (E-selectin, $27.3 \pm 5.40 \mathrm{RFU}$ vs $\mathrm{LacZ}, 45.5 \pm 7.37 \mathrm{RFU}, \mathrm{p}=0.013$ ) or no siRNA group (E-selectin, $27.3 \pm 5.40 \mathrm{RFU}$ vs no siRNA, $52.5 \pm 10.4$ RFU, $\mathrm{p}=0.010)$.

\subsection{Effect of Gene Silencing of E-Selectin on EPC Adhesion to HUVEC under Flow}

Figure 5 showed results of the flow assay using IL-1activated HUVECs after siRNA transfection. There was

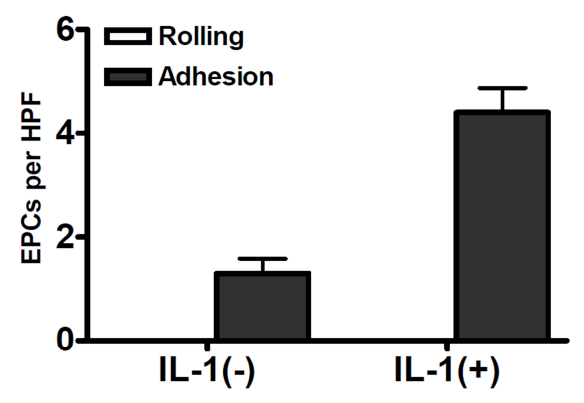

Figure 3. The number of rolling and adherent EPC to activated HUVEC were measured as described in Methods. $\left(^{*}=\mathrm{p}<0.05\right.$ vs $0 \mathrm{~h}$ IL-1 $)$; data based on 10 fields of observation for each condition. EPCs were perfused over a HUVEC monolayer (grey background) that had been subjected to activation by IL- $1 \beta(50 \mathrm{U} / \mathrm{mL})$ for two different time periods $(0 \mathrm{~h} \mathrm{IL}-1 \beta$ and $4 \mathrm{~h} \mathrm{IL}-1 \beta)$.

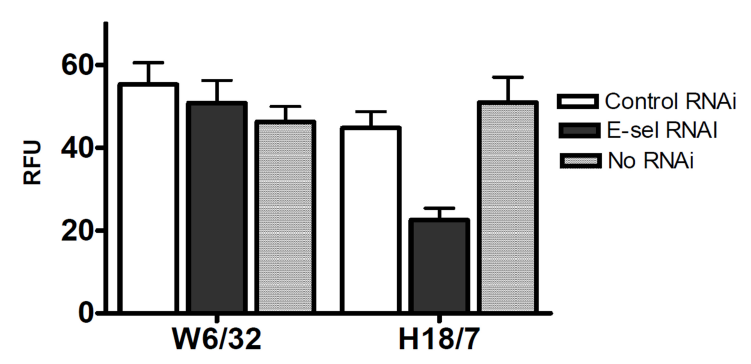

Figure 4. Cell surface expression of E-selectin (H18/7) and positive control (W6/32) in HUVEC after siRNA silencing. The expression intensity of each of the adhesion molecules was determined using a fluorescent immunobinding assay as described in Methods. $*^{*}=\mathrm{p}<0.05$ vs corresponding 0hr IL-1 $)$; data based on 5 repeats for each condition.

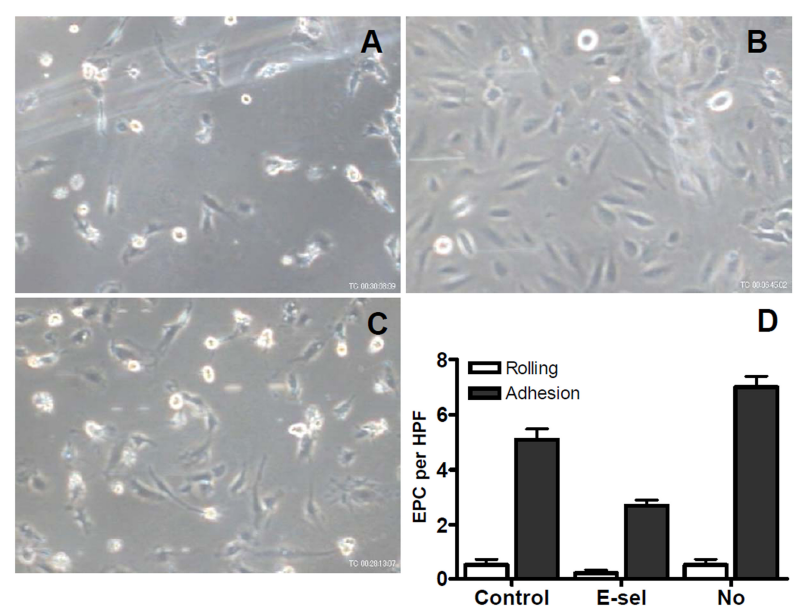

Figure 5. The number of rolling and adherent EPC to activated HUVEC after siRNA transduction. Graph showing the effect of E-selectin siRNA transfection on rolling and adhesion of EPCs compared to control siRNA transfection and no siRNA treatment. (* $=\mathrm{p}<0.05$ vs control); data based on 10 fields of observation for each condition.

a significant decrease in EPC adhesion in HUVECs transfected with E-selectin siRNA compared to those treated with $L a c Z$ siRNA (controls) (E-selectin siRNA, $2.7 \pm 0.64$ cells vs $L a c Z$ siRNA, $5.1 \pm 1.22$ cells, $\mathrm{p}=$ $\left.5.74 \times 10^{-5}\right)$. In comparison, there was no significant difference in rolling between those HUVECs transfected with E-selectin siRNA and the control HUVECs (Eselectin siRNA, $0.2 \pm 0.4$ cells vs $L a c Z$ siRNA, $0.5 \pm$ 0.67 cells, $p=0.26$ ). There was also a significant decrease in EPC adhesion in HUVECs treated with E-selectin siRNA compared to those treated with no siRNA (Lipofectin ${ }^{\circledR}$ only) (E-selectin siRNA $2.7 \pm 0.64$ cells vs no siRNA, $7 \pm 1.26$ cells, $\mathrm{p}=3.74 \times 10^{-8}$ ).

\section{DISCUSSION}

In this project, we have demonstrated that 1) $4 \mathrm{~h}$ of in- 
cubation with IL-1 $\beta$ induces adhesion of HL60 cells to HUVEC under flow; 2) $4 \mathrm{~h}$ of incubation with IL-1 $\beta$ induces expression of E-selectin and ICAM-1 in HUVEC; 3) $4 \mathrm{~h}$ of incubation with IL-1 $\beta$ induces adhesion of EPCs to HUVEC under flow; 4) siRNA against E-selectin reduces endogenous expression of E-selectin in HUVEC; 5) gene silencing of E-selectin resulted in reduction of EPC adhesion to HUVEC. These lines of findings point a predominant role for this molecule in neovascularisation. In previous work from our laboratory using siRNA transfection carried out by Nishiwaki et al (2003), we successfully silenced endogenous Eselectin expression in vascular endothelium using siRNA transfection, and demonstrated that this inhibition caused a significant reduction in leukocyte adhesion [8]. Current results compliment this work and emphasise the efficacy of siRNA in the field of vascular biology.

We have also reinforced the work of Nishiwaki et al (using antibodies to downregulate E-selectin) and Vajkoczy et al (using an in vivo method to silence Eselectin expression) in demonstrating that E-selectin is important in EPC adhesion by using a different method (siRNA transfection) $[6,7]$.

Using siRNA transfection to inhibit E-selectin expression, as demonstrated by our experiments, may have implications for its role in angiogenesis. Bischoff has reported that E-selectin is involved in angiogenesis through a series of experiments [17]. One of these experiments showed that antibodies directed against Eselectin or sialylated fucosylated oligosaccharides (structures to which E-selectin binds) inhibit the formation of capillary-like tubes in vitro [18]. The presence of Eselectin in human infantile haemangioma tumours (endothelial cell tumour of capillary blood vessels), as well as in tissues with on going growth of microvessels (such as human placenta and neonatal foreskin) has been shown in vivo [19]. Yu et al had also shown that endothelial progenitor cells are present in infantile haemangioma; 11 of 12 proliferating haemangioma specimens contained EPCs (determined by flow cytometry using CD133 and CD34 as markers for EPCs) [20]. This raises the possibility of using our method of silencing Eselectin expression in treating tumour angiogenesis, by preventing localised EPC adhesion to the endothelium, thus inhibiting angiogenesis in the tumour, reducing its growth. As described previously, we found that there was no significant reduction in rolling after E-selectin down-regulation. This could have been because of the low level of EPC rolling in general, as demonstrated in Figure 3. On the other hand, the binding characteristics of E-selectin (E-selectin has a greater role in adhesion than rolling) may have some effects. This speculation has been supported by the results from Mazo, et al.'s experiments [21]. They demonstrated that blocking Eselectin with antibodies resulted in a reduction of haematopoietic progenitor cells (HPC) rolling of $32 \%$ compared to a reduction in HPC rolling of $58 \%$ when P-selectin was inhibited (measured using intravital microscopy). Therefore the other selectins ( $\mathrm{P}$ - and Lselectins) are probably more important for HPC rolling.

A limitation of this experiment is that we have only demonstrated that gene silencing of E-selectin expression reduces EPC adhesion in vitro, but not in vivo. Therefore future work could be aimed at seeing if siRNA transfection has similar effects in vivo. It would also be interesting to try similar experiments using siRNA transfection against other adhesion molecules such as ICAM1 or P-selectin, and look at the effects on EPC recruitment.

In conclusion, we have demonstrated that E-selectinshort interfering RNA inhibits endothelial progenitor cell recruitment to vascular endothelium under flow conditions.

\section{ACKNOWLEDGEMENTS}

Grateful thanks to Dr Naokazu Nakamura, Michiyo Deushi, Noriko Nitta and Mariko Tani for their experimental help and advice, Professor Peter Sugden for his help and advice throughout the course of the project.

\section{REFERENCES}

[1] Cines, D.B., Pollak, E.S., Buck, C.A., Loscalzo, J, Zimmerman, G.A., McEver, R.P., Pober, J.S., Wick, T.M., Konkle, B.A., Schwartz, B.S., Barnathan, E.S., McCrae, K.R., Hug, B.A., Schmidt, A.M. and Stern, D.M. (1998) Endothelial cells in physiology and in the pathophysiology of vascular disorders. Blood, 91(10), 3527-3561.

[2] Shi, Q., Rafii, S., Wu, M.H., Wijelath, E.S., Yu, C., Ishida, A., Fujita, Y., Kothari, S., Mohle, R., Sauvage, L.R., Moore, M.A., Storb, R.F. and Hammond, W.P. (1998) Evidence for circulating bone marrow-derived endothelial cells. Blood, 92(2), 362-367.

[3] Kakkar, A.K. and Lefer, D.J. (2004) Leukocyte and endothelial adhesion molecule studies in knockout mice. Current Opinion in Pharmacology, 4(2), 154-158.

[4] Muller, W.A. (2003) Leukocyte - endothelial-cell interactions in leukocyte transmigration and the inflammatory response. Trends in Immunology, 24(6), 326-333.

[5] Chavakis, E., Aicher, A., Heeschen, C., Sasaki, K., Kaiser, R., El Makhfi, N., Urbich, C., Peters, T., ScharffetterKochanek, K., Zeiher, A.M., Chavakis, T. and Dimmeler, S. (2005) Role of beta2-integrins for homing and neovascularization capacity of endothelial progenitor cells. Journal of Experimental Medicine, 201(1), 63-72.

[6] Vajkoczy, P., Blum, S., Lamparter, M., Mailhammer, R., Erber, R., Engelhardt, B., Vestweber, D. and Hatzopoulos, A.K. (2003) Multistep nature of microvascular recruitment of ex vivo-expanded embryonic endothelial progenitor cells during tumor angiogenesis. Journal of Ex- 
perimental Medicine, 197(12), 1755-1765.

[7] Nishiwaki, Y., Yoshida, M., Masuda, H. and Isobe, M. (2004) Recruitment of bone marrow-derived endothelial progenitor cells to vascular endothelium involves E-selectin dependent mechanism. Cardiovascular Pathology, 13(3), 171.

[8] Nishiwaki, Y., Yokota, T., Hiraoka, M., Miyagishi, M., Taira, K., Isobe, M., Mizusawa, H. and Yoshida, M. (2003) Introduction of short interfering RNA to silence endogenous E-selectin in vascular endothelium leads to successful inhibition of leukocyte adhesion. Biochemical and Biophysical Research Communications, 310(4), 1062-1066.

[9] Yoshida, M. and Gimbrone, M.A. Jr. (1997) Novel roles for E-selectin in endothelial-leukocyte adhesion. Annals of the New York Academic Sciences, 811, 493-497.

[10] Yoshida, M., Szente, B.E., Kiely, J.M., Rosenzweig, A. and Gimbrone, M. Jr. (1998) Phosphorylation of the cytoplasmic domain of E-selectin is regulated during leukocyte-endothelial adhesion. The Journal of Immunology, 161(2), 933-941.

[11] Bevilacqua, M.P., Pober, J.S., Mendrick, D.L., Cotran, R.S. and Gimbrone, M.A. (1987) Identification of an inducible endothelial-leukocyte adhesion molecule. Proceedings of the National Academy Sciences of USA, 84(24), 9238-9242.

[12] Luscinskas, F.W., Cybulsky, M.I., Kiely, J.M., Peckins, C.S., Davis, V.M. and Gimbrone, M.A. (1991) Cytokineactivated human endothelial monolayers support enhanced neutrophil transmigration via a mechanism involving both endothelial-leukocyte adhesion molecule-1 and intercellular adhesion molecule-1. The Journal of Immunology, 146(5), 1617-1625.

[13] Gerszten, R.E., Garcia-Zepeda, E.A., Lim, Y.C., Yoshida, M., Ding, H.A., Gimbrone, M. Jr., Luster, A.D., Luscinskas, F.W. and Rosenzweig, A. (1999) MCP-1 and IL-8 trigger firm adhesion of monocytes to vascular endothe- lium under flow conditions. Nature, 398(6729), 718-723.

[14] Kiely, J.M., Luscinkas, F.W. and Gimbrone, M. Jr. (1999) Leukocyte-endothelial monolayer adhesion assay (static conditions). Methods in Molecular Biology, 96, 131-136.

[15] Gerszten, R.E., Luscinskas, F.W., Ding, H.T., Dichek, D.A., Stoolman, L.M., Gimbrone, M.A. Jr. and Rosenzweig, A. (1996) Adhesion of memory lymphocytes to vascular cell adhesion molecule-1-transduced human vascular endothelial cells under simulated physiological flow conditions in vitro. Circulation Research, 79(6), 1205-1215.

[16] Luscinskas, F.W., Kansas, G.S., Ding, H., Pizcueta, P., Schleiffenbaum, B.E., Tedder, T.F. and Gimbrone, M. Jr. (1994) Monocyte rolling, arrest and spreading on IL-4activated vascular endothelium under flow is mediated via sequential action of L-selectin, $\beta 1$-integrins, and ß2-integrins. Journal of Cell Biology, 125(6), 1417-1427.

[17] Bischoff, J. (1997) Cell adhesion and angiogenesis. Journal of Clinical Investigation, 99(3), 373-376.

[18] Nguyen, M., Strubel, N.A. and Bischoff, J. (1993) A role for sialyl Lewis-X/A glycoconjugates in capillary morphogenesis. Nature, 365(6443), 267-269.

[19] Kraling, B.M., Razon, M.J., Boon, L.M., Zurakowski, D., Seachord, C., Darveau, R.P., Mulliken, J.B., Corless, C.L. and Bischoff, J. (1996) E-selectin is present in proliferating endothelial cells in human hemangiomas. American Journal of Pathology, 148(4), 1181-1191.

[20] Yu, Y., Flint, A.F., Mulliken, J.B., Wu, J.K. and Bischoff, J. (2004) Endothelial progenitor cells in infantile hemangioma. Blood, 103(4), 1373-1375.

[21] Mazo, I.B., Gutierrez-Ramos, J.C., Frenette, P.S., Hynes, R.O., Wagner, D.D. and von Andrian, U.H. (1998) Hematopoietic progenitor cell rolling in bone marrow microvessels: parallel contributions by endothelial selectins and vascular cell adhesion molecule 1. Journal of Experimental Medicine, 188(3), 465-474. 